\title{
LES MALHEURS DE KUNNANITYA, FEMME DE MARCHAND
}

C. MICHEL

Parmi les très nombreuses lettres paléo-assyriennes retrouvées dans le kārum de Kaniš, la correspondance féminine occupe une place non négligeable'. D'une part, lors de la création des comptoirs commerciaux, les marchands assyriens qui partent exercer leur métier en Anatolie laissent à Aššur leurs épouses et leurs enfants en bas âge; celles-ci, en charge de la gestion de leur maison à Aššur, écrivent fréquemment à leurs maris. D'autre part, quelques femmes, vivant auprès de leurs époux à Kaniš ou dans d'autres localités anatoliennes, ont également laissé des missives, échangées avec des membres de leur famille. Les femmes assyriennes sont de loin les mieux représentées au sein de cette correspondance féminine, et plusieurs d'entre elles ont inspiré différentes publications ${ }^{2}$. En revanche, la vie et les préoccupations

1. Une liste des femmes avec leur ville de résidence et, dans la mesure du possible, leurs relations familiales est offerte par K. Hecker, "tib'immo atalkimAssyrerinnen im karumzeitlichen Anatolien", ArOr 47 [1979], 404-418. Un échantillon de la correspondance féminine est présenté par K.R. Veenhof, "Brieven van Assyrische Vrouwen uit de Correspondentie der Oudassyrische Handelaars", Schrijvend Verleden Documenten uit het oude Nabije oosten Vertlaad en toegelieht, Mededelingen en Verhandelingen, Leiden 1983, ("Brieven") 81-93.

2. K.R. Veenhof, AOATT, 103-123, P.Garelli, "Femmes d'affaire en Assyrie", Aror 47 [1979], 42-49 (Tarišmātum et Ištar-Lammassi), C. Günbatt, “Some observations about the commercial activities of women in the light of the Kültepe Tablets", Hittite and other Anatolian and Near Eastern Studies in Honour of Sedat Alp, Ankara 1992, (Mél, S_Alp) 229-234 (Waqqurtum, Lamassī, Ištar-bāštī et Nuhšatum), K. Lyczkowska, "Some Data about Subultum", dans B. Hruska et G. Komoroczy (éds.), Festschrift Lubor Matous, Budapest 1978, 131-137 ou encore L. Matous, "Zur Korrespondenz des Indi-lim mit Tarām-kubi", Zikir sumim: Assyriological Studies Presented to F.R. Kraus on the Occasion of his Seventieth Birthday, Leiden 1982, 268-270. 
de leurs homologues anatoliennes, plus rares dans les textes, restent encore mal connues. Kunnaniya, habitante de Kaniš, s'est tout naturellement imposée par l'abondance de sa correspondances-une petite quinzaine de lettres-, et la richesse de son contenu. Il m'est donc particulièrement agréable d'offrir cet article sur la correspondance d'une femme anatolienne au Professeur E. Bilgiç, l'auteur d'une étude majeure sur la terminologie indigène dans les tablettes des marchands paléo-assyriens de Kaniš.

\section{Les attestations de Kunnaniya dans les textes paléo-assyriens}

Kunnaniya porte un nom vraisembslablement anatolien qui serait formé des éléments hittites kunna-: "droit" et anni-: "mère"3. Une telle interprétation indiquerait qu'il s'agit d'un nom strictement féminin ${ }^{4}$.

Hormis les quatorze lettres appartenant à la correspondance de Kunnanīya, ce nom propre intervient dans une demi-douzaine de

3. E. Laroche, Les Noms des Hittifes, 98, A. Goetze, JCS 9 [1954], 77, note 58 et P. Garelli, $A C, 144$.

4. Or, si effectivement la plupart de nos références renvoient à une femme, deux textes feraient plutôt allusion à un nom porté par un homme. Le document juridique $I C K$ 1, 5, 10-11 mentionne peut-être Kunnaniya, fils d'Ikua, 11. 5-12: "Ces hommes, Laqēpum les produira pour témoignage comme quoi l'argent (appartient) à Kunnaniya, fils d'Ikua" a-wi-li: a-ni-ú-tim, Lá-qé-pu-um, a-na ši-bu-tim, ú-šé-lá-šu-nu, ki-ma: KÙ.BABBAR, (érasure de a-ma) ša $K u$-na-ni-a/"ni", DUMU $I-k u-a$, ( érasure), et la reconnaissance de dette inédite Prague I 594, 3, relatant une dette de Kunnaniya envers Laqēpum, fils de Sabahanum, formule la clause de remboursement à la troisième personne du masculin singulier: "Laqëpum, fils de S̆abahanum, a en créance 6 mines d'argent sur Kunnaniya (...) il remboursera ( $i$-sa-qay) dans 5 mois". Ce dernier texte m'a êté communiqué par $\mathrm{K}$. Hecker. L'existence d'un nom masculin Kunnanyya pourrait avoir pour origine la synthèse entre Kunnaniya (fém). et Kunnananiya formé des éléments kunna- "droit" et naniya "frère" (masc.) Par ailleurs, Kunnaniya apparaît comme témoin à deux reprises, au sein de listes de témoins masculins (ICK 1, 112, 15 et TC 2,72,16=EL 158). Ces deux textes qui $n$ 'apportent aucune information sur la personnalité de Kunnantya sont sans doute à rapprocher de la tablette de Prague. En effet. le premier de ces deux textes, ICK 1, 112, concerne une dette de Laqëpum, fils de Šabahanum, le créancier de Kunnanīya dans le document Prague I 594. 
tablettes juridiques; deux d'entre elles, qui n'ont aucun lien apparent avec la correspondance de Kunnaniya, sont des contrats d'achat de personne'. Le premier concerne l'acquisition par Ahātum d'une esclave auprès de Kunnaniya et Nakišduar ( $B I N$ 4, 183). J. Lewy, qui a édité cette tablette, suppose qu'il s'agit de la vente d'une enfant par ses parents, et il sous-entend donc que Kunnanĩya est l'épouse de Nakišduar, autre Anatolien au nom hittite ${ }^{6}$. Le second, en revanche, relate la vente de Kunnanīya, pour la modique somme de 15 sicles d'argent, par Šihišnuman et la mère de la jeune fille?. Il est probable que, dans ce contrat, Kunnanìya soit plutôt placée en gage auprès des créanciers de ses parents.

Les documents juridiques mis à part, Kunnaniya intervient dans les formules épistolaires de quatorze lettres; à dix reprises en tant que destinataire, et quatre fois comme expéditrice ${ }^{8}$ :

En-têtes des lettres de la correspondance de Kunnanya
1. $B I N 6,1$
d' Ašsur-muttabbil
2. $\mathrm{KTH} 6$
d' Ašsur-muttabbil
3. C Kalley 36
d' Asšur-muttabbil
4. $K T S 2,31$
d' Aşur-muttabbil
5. 1. $B I N 6,84$
d' Assur-muttabbil

6. $K T B I 5$

d' Ašsur-muttabbil à Kunnañya

à Kunnanĩya

à Kunnanĩya

à Kunnaniya

à Karunuwa et

Kunnaniya

à Nimar-Ištar,

Kunnaniya,

Šamaš-taklaku et

Sulliya

7. CTMMA 1,78 à (Irma-Aššrur), Walhišna et Kunnaniya d’ Ašsur-muttabbil

5. Les autres documents sont mentionnés dans la note précédente.

6. Cf. EL 214 et le commentaire $E L$ 1, p.204. Notons que cette hypothèse implique que la femme, Kunnaniya, est mentionnée avant son époux.

7. Tablette Kt Ankara 14-1-80 publiée par C. Günbatt, "Ankara Anadolu Medeniyetleri Müzesi'nde bulunan üç tablet", Cumhuriyetin 60. Yıldönümü̈ armağanı Ankara 1987, 189-199, texte 2.

8. La tablette $A K T 1,14$ a été éditée une première fois par le dédicataire de cet articie, E. Bilgiç, Die Einheimischen Appellativa der Kappadokischen Texte und ihre Bedeutung für die Anatolischen Sprachen, DTCFY 96, Ankara 1954, 59 sous la cote Ank. 14. La transcription par B. Landsberger du texte inédit $C$ Kalley 36 m'a été communiquée par K.R. Veenhof. 


\begin{tabular}{|c|c|c|}
\hline 8. HUCA 40,69 & de Walhišna & à Kunnanīya \\
\hline 9. $C C T 4,21 \mathrm{a}$ & d' Ennam-Ašsur & $\begin{array}{l}\text { à Kunnaniya et } \\
\text { Alăhnum }\end{array}$ \\
\hline 10. BIN 6,17 & à Kunnaniya & d' Aššur-taklāku \\
\hline 11. $A K T 1,14$ & à Šeșur & de Kunnanĩya \\
\hline 12. $T T C 26$ & à Asu(w)elka et Adue & $\begin{array}{l}\text { de Kunnaniya et } \\
\text { Sāt-Aššur }\end{array}$ \\
\hline 13. $K T H 5$ & à Asu(w)elka & de Kunnaniya \\
\hline 14. $C C T 6,7 \mathrm{~b}$ & à Kubinahšsu & de Kunnaniya \\
\hline
\end{tabular}

\section{La famile de Kunnaniya}

Malgré le nombre relativement restreint de missives appartenant à la correspondance de Kunnaniya, ce personnage a suscité plusieurs notes prosopographiques en désaccord les unes avec les autres. Selon K. Hecker, il existerait deux homonymes de ce nom'. L'une serait la femme d'Aššrur-ennam, tandis que la seconde aurait épousé Aššur-muttabbil. Il est reconnu que ces deux hommes font partie des proches de Kunnaniya.W.G. Gwaltney, dans son édition des tablettes conservées à Philadelphie, propose de voir en Aššur-ennam le mari de Kunnaniya et en Walha/išna, son frère ${ }^{10}$. Il ne prend cependant pas parti sur l'existence d'une homonyme de Kunnaniya. La publication de textes conservés à Istanbul par V. Donbaz, et de ceux de New York par M.T. Larsen, permet de montrer que cette correspondance relève en fait d'une seule femme. En effet, non seulement la missive $K T S$ 2, 31 adressée par Ašsur-muttabbil à Kunnaniya, fait mention, 1.21, d'huile appartenant à Walbišna", mais surtout, la lettre CTMMA 1 ,

9. K. Hecher, ArOr 47 [1979] 112.

10. W.G. Gwaltney, HUCA sup 3 [1983], texte 42, p. 100-101, édition de la lettre HUCA 40, 69. Notons que sa traduction du terme Emum par "beau-frère" est confirmée par K.R. Veenhof, lorsqu'il recense une quinzaine de mariages mixtes entre Assyriens et Anatoliens, parmi lesquels celui de Kunnaníya et d'Ašsur-ennam, "The Old Assyrian Merchants and their Relations with the Native Population of Anatolia", BBVO 1, 158, note 19.

11. Texte 4=KTS 2, 31, 19-23: "Transforme et envoie-moi - litre d'huile de Kanišs sur l'huile de Walbišna." - SìLa I.GIŠ: sa Kà-ni-iš, [i]-na ša-am-ni-im, ša Wa-al'-his-iš-na, (*) šu-pá-i-li-ma, šé-bi $i_{4}$ lim. La ligne 22 omise par V. Donbaz est due à W. Farber, Aula Or. 8 [1990], 143. 
78 est expédiée par Aššur-muttabbil à Walhišna et Kunnaniya. V. Donbaz présente les correspondants de la lettre KTS 2, 31, Aššr-muttabbil et Kunnaniya, comme étant mari et femme, et dans son commentaire de la 1.21, il mentionne Walhišna en tant que frère de Kunnaniya ${ }^{12}$. K. Nashef montre l'ambivalence de l'anthroponyme Walha/išna, et il prouve que, dans le cas présent, Walhišna est une femme, soeur de Kunnaniy $a^{13}$. Ces deux nouvelles missives, publiées il y a moins de dix ans, qui s'ajoutent aux cinq autres échangées entre Aššur-muttabbil et Kunnanīya incitèrent quelques auteurs à confirmer le lien matrimonial entre Kunnaniya et Aššur-muttabbil en dépit du document de Philadelphie selon lequel Kunnaniya pourrait être l'épouse d'Aššur-ennam ${ }^{14}$. D'autres enfin, tentèrent de trouver un compromis en tentant de marier Kunnaniya à l'un et l'autre marchand assyrien successivement ${ }^{15}$ ! Ces différentes interprétations montrent parfaitement la complexité de la situation crée par ces quelques lettres.

La récurrence de plusieurs acteurs dans cette correspondance indique clairement l'appartenance de ces missives à une seule

12. Il confirme le lien unissant Walha/îsna à Kunnanīya dans "The Business of Ašèd, an Anatolian Merchant", AfO 35 [1988], 57.

13. K. Nashef, Recension de KTS 2, WO 24 [1993], 169-170 (à propos du texte KTS 2, 31, 21). Notons cependant que ce demier affirme des relations familiales contradictoires entre les différents personnages. Refusant une traduction d'emum par "beau-frère", il traduit le terme par "beau-père". Faisant de Walhišna et Kunnaniya deux soeurs, et d'Ašsur-ennam le beau-père de la première, il conclut au mariage entre Kunnaniya et Aššr-ennam! K. Nashef ajoute que, selon lui, le mari de Walhišna serait Irma-Aššr (codestinataire de CTMMA 1, 78). Mais sa correction de la 1.8 de ce texte n'est pas satisfaisante, cf. K. Hecher, NABU 1990/139. Le sexe féminin de Walhnišna dans notre dossier est effectivement révélé par les pronoms enclitiques féminins recensés dans la missive CTMMA 1, 78 qui lui est adressée. Les autres mentions de cet anthroponyme dans la documentation paléo-assyrienne renvoient à un homme, père d'Alulū $K t n k$ 73,4-5 et 74,3, époux d'Amakani et père d'"Elălí $K t n / k$ 1888, 12, textes cités par V. Donbaz, AfO 35 [1988], 191a, ou encore frère de Pithana, et fils de Galủa et Asunina, $K t v) k$, 28, 3. C. Günbatt, Belleten 53 [1989], 55.

14. K.R. Veenhof, $N A B U 1992 / 5$, à propos de $K T H 6$.

15. Comme par exemple W.G. Gwaltney, dans sa recension de V. Donbaz, KTS 2, JAOS 112 [1992], 334. 
femme, Kunnaniya ${ }^{16}$. Celle-ci, de par le contenu des lettres qu'elle échange avec Aššur-muttabbil est vraisemblablement son épouse. Or, bien qu'il existe une quinzaine d'homonymes pour ce nom masculin, le mari probable de Kunnaniya est identifié par son sceau apposé sur l'enveloppe CTMMA $1,78 \mathrm{c}$ au fils du célèbre marchand de Kaniš, Pūšu-kēnn ${ }^{17}$. Dès lors que l'on suppose Kunnaniya mariée à Aššur-muttabbil, il convient de règler le cas d'Aššur-ennam, l'hypothétique époux de Kunnanīya selon l'interprétation du terme emum dans la lettre expédiée par Walhišña ${ }^{18}$ :

"Dis à Ašsur-ennam: (...) tu (es) mon emum. Si tu abandonnes ma soeur, tu verras ce que je ferai de toi!"

La traduction du mot emum est controversée; certains contextes imposent une traduction par "gendre", d'autres impliqueraient celle de "beau-père"20. En réalité, il semble que ce terme traduise essentiellement une relation familiale par alliance, c'est pourquoi plusieurs auteurs ont admis la traduction de

16. Ainsi les sept premières lettres forment un lot cohérent car elles correspondent aux échanges épistolaires entre Kunnaniya et Aššrur-muttabbil, la lettre 8 se rattache au même dossier par la présence de Walhišna, Les missives 11, 12 et 13 concernent Adue, Asu(w)elka, Šat-Aššur et Šẹșur, et grossissent le dossier précédent par la mention des fils d'Aššur-muttabbil (11). Restent trois messages, l'un, 14, trop fragmentaire, $n$ 'apporte aucune information, tandis que les deux autres 9 et 10, par quelques allusions, peuvent être rapprochés des autres lettres.

17. B. Tessier, Sealing and Seals on Texts from Kültepe Karum Level 2, Istanbul 1994, 201, le sceau n 634 apposé sur CTMMA 1,78c est identique à celui d'Ašssur-muttabbil, fils de Pūsu-kēn, sceau E de l'enveloppe ATHE 24b. Ce lien matrimonial pourrait être confirmé par la lettre 11. En effet, dans cette missive, Kunnaniya estime que le loyer d'une maison qui lui est dû n'a pas été reçu par les fils d'Aššrur-muttabbil (comme cela aurait été normal) mais par un de leurs cousins (cf. ci-dessous).

18. Texte $8=$ HUCA $40,69,8-9$ et 14-18 lettre adressée par Walhyišna à Kunnaniya, a-na A-šur-e-na-fam, qi-bi $i_{4}-m a$ (...) e-mi: a-ta, šu-ma a-ha-ti, ¿é-zi-ib, ša e-pu-šu-kà, ıa-mar.

19. Par exemple VS 26, 64 et le commentaire de K.R. Veenhof, p. 23 ou encore CCT 6, 11 a, 32-39.

20. EL 187, 10 ou encore BIN 6, 183, 8 dans l'expression bît emi. 
"beau-frère" dans le texte 8. Exceptée cette lettre, Aššur-ennam n'apparaît qu'une seule fois dans notre dossier, parmi d'autres transporteurs subalternes dans le document $\mathbf{5}$ envoyé par Aššur-muttabbil à Karunuwa et Kunnaniya ${ }^{21}$ :

“Aššsur-ennam t'a apporté 3 sicles d'argent et u[ne étril]le?."

Qu'Ǎ̌šur-ennam soit un gendre de Walhił̌na, peut-être fils d'Aššur-muttabbil, ou encore son beau-père, chargé de veiller sur les intérêts de Kunnaniya, importe finalement peu pour notre propos étant donné son rôle secondaire dans ce clossier. En revanche, il paraît intéressant de noter que, selon les formules épistolaires, Walhišna serait non seulement plus âgée que sa soeur envers laquelle elle fait preuve d'une attention presque maternelle (8), mais également que son beau-frère, Aššsur-muttabbil.

La correspondance de Kunnaniya révèle deux phases dans la vie de cette femme. Les lettres qu'elle échange avec son mari reflètent les activités classiques d'une femme de marchand installée à Kaniš et qui participe activement à l'entreprise familiale, tandis que la majorité des autres lettres du dossier, caractérisée par l'absence de la mention d'Aššr-muttabbil, offrent l'image d'une femme abandonnée, confrontée à des conflits familiaux et passablement démunie.

\section{Kunnaniya, femme d'Assur-muttabbil}

Kunnaniya réside à Kaniš où son mari demeure également en temps normal ${ }^{22}$. Toutefois, ce dernier voyage souvent, que ce soit

21. Texte $5=B I N 6,84,7-8: 3$ GÍN KÙ.BABBAR [1 pá-na]-ra-am, A-šur-e-n [am $u b$-lá]ki-im.

22. Notre dossier est sans doute postérieur à la mort de Pứsu-kēn, puisque l'on y voit Buzāzu et Aššur-muttabbil se disputer la propriété de servantes sans doute issues de I'héritage paternel (7). or les différentes lettres envoyées par Ahaha à ses frères ( $C C T 58 \mathrm{a}, T C 2,46)$ et plus particulièrement adressées Aššur-muttabbil (KTH 7) sont expédiés à Kannišs. Pour la famille de Pứsu-kēn, cf. J. Lewy, HUCA 27 [1956], 79, note 333. 
en Anatolie ou à Ašsur $(5,6)$ et les instructions qu'il envoie à plusieurs reprises à Kunnanìya témoignent de son absence, certaines annonçant son arrivée iminente ${ }^{23}$ :

“Achète dix sacs (de céréales), pour moitié de blé, afin qu'ils soient disponibles à mon arrivée"

Profitant de ses nombreux voyages, Ašsur-muttabbil approvisionne Kunnaniya. Depuis Aššur, il lui fait parvenir étain et étoffes ${ }^{24}$, et toutes sortes d'objets hétéroclites de ses différents périples $^{25}$. Certains de ces envois sont assurés par des transporteurs ou des serviteurs du couple ${ }^{26}$. Parmi eux, Karunuwa, frère d'Alili, apparaît à trois reprises. Chargé dans un premier temps d'apporter une étoffe pour vêtir un enfant, cinq coupes originaires de Habura et deux sicles d'argent, il arrive finalement chez Kunnaniya muni de l'étoffe et des coupes, mais l'argent a été converti en $15-$ sicles d'étain ${ }^{27}$.

23. Texte 3=C Kalley, 36, 14-18: 10 na-uru-uq mi-ǐ̀-lam, ar-ša-tim, ša-a-mì-ma, li-bi $i_{4}-\tilde{s} i$, a-dí e-ra-ba-ni.

24. Texte $3=$ C Kalley 36, 3-8: "Ennum-i 1 , fils de Kura, $t$ 'apporte 10 sicles d'étain à mon sceau avec Ahuni...", 10 GiN AN.NA, ku-nu-ki-a, En-um-i-li DUMU Ku-ra, iš-rí A-hu-ni, wa-áś-bu? [x]-tim, na-(ás-a)-ki-im.

25. Dans le texte $5=B I N 6,84$, Kunnanīya reçoit de son mari de nombreuses objets: étrilles, laine, étoffes, châles, balais?, miroir et d'autres non identifiés, ainsi que de l'huile. Ces différents objets proviennent de Zalpa, Aššur et d'autres localités de Syrie du nord ou d'Anatolie.

26. Texte $4=K T S 231,23,24$ : "Les serviteurs ne doivent pas passer une seule nuit (là-bas), qu'ils viennet ici”, sú-ha-ru, ú-ma-kál: lá i-be-tù, lí-tal-ku-ni-im. Voir egalement le texte $6=K T B I$ 5, 21-24: "Dis à Nimar-Istar:que ton rapport me parvienne pour me dire si, là-bas, les serviteurs sont retardés," a-na

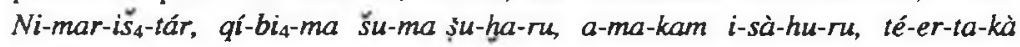
li-li-kam.

27. Texte 3= C Kalley 36, 8-13: "Karunuwa, le frère d'Alili, doit t'apporter une étoffe de la petite, 2 sicles d'argent (et) 5 coupes”, 1 TÚG śa șu-ha-ar-tim, 2 GíN KÙ.BABBAR 5 kà-sà-tim, Kà-ru-nu-wa a-hu-tí, A-li-li, li-di-na-ki-im. Texte 4= KTS 2 31, 3-11: "Je lui (à Karunuwa) ai donné une étoffe pour vêtir l'enfant et 15 - sicles d'étain à la place de 2 sicles d'argent. Karunuwa, frère d'Alili, t’a apporté 5 coupes de Habura.", 1 TÚG sa șu-ùh-ri-im ù, 15 - GÍN KÙ.BABBAR a-dí-su-um, 5 kà-sà-tim, ša Ha-bu-ra-a, Kà-ru-nu-wa-a, a-hu-ú: $A$-li-li, ub-lá-ki-im. Voir également le texte $5=B I N 684,30-34$, lettre adressée 
En échange, Ašsur-muttabbil demande occasionnellement à Kunnanīya de lui faire parvenir des denrées locales comme de l'huile de Kanišs ${ }^{28}$. En l'absence de son époux, Kunnanīya gère sa maisonnée. Parmi ses multiples activités à Kanišs, l'une, particulièrement originale dans les tablettes paléo-assyriennes, consiste en l'élevage de porcs; Ašsur-muttabbil lui donne des directives à ce sujet ${ }^{29}$ :

"Si les porcs n'engraissent pas, vends-les, s'ils sont gras, qu'ils soient disponibles."

Par ailleurs, Kunnanīya assure l'intendance par l'achat de provisions pour les serviteurs ${ }^{30}$, et à plusieurs reprises, son époux lui demande de prendre soin de Šãt-Aššur, une enfant dont le statut n'est pas clair, mais qui est vraisemblablement à la charge du

à Kanunuwa et Kunnaniya: "Karunuwa, le frère d'Alili, t'a apporté une étoffe pour vêtir l'enfant.", 1 TÚG! ša lu-bu-歺-im, ša șu-ùh-ri-im, Kà-ru-nu-wa: $a-h u, A-l i-l i, u b-l a ́-[k i-i m]$.

28. Texte 4=KTS 2, 31, 19-23, ci-dessus, note 11 , ou encore le texte $\mathbf{2}=\boldsymbol{K} T H \mathbf{6}$, 22-24: "Envoie-moi de l'huile fine...", İ.GIŠ SIGs..., Še-bid-lim.

29. Texte $5=B I N \quad 6$ 84, 35-37: šsu-ma hu-zi-ru: lá-i-kà-b[e-ru], a-ši-mì-im: dí-na-šsu-nu su-ma, kà-be-ru: li-zi-zu. Pour les élevages de porcs à Kaniš, cf. C. Michel, "A table avec les marchands paléo-assyriens", CRRAI 39, Heidelberg 1992 (à paraitre).

30. Texte $2=K T H 6,2-9$ : "Achète pour les serviteurs $\frac{1}{2}$ litre de cumin, 2 fagots de şumlālum pour un montant d'un sicle d'argent, des siparrātum, 30 sacs et 3 étoffes-menunianum d'un sicle d'argent pièce, et mets-toi en route." $\frac{1}{2}$ SílAA, kà-mu-ni 2 ri-ik-sí, sú!-um-Lá-le-e ša 1 GíN, KÙ.BABBAR șí-pá-ra-tim, 30 $i$-lá-tim 3 TÚG me-nu-ni-a-/ni, ša 1 GÍN.TA KÙ.BABBAR (érasure), a-şu-ha-ri ša-a-me-ma, té-eb-e-ma: a-tal-ki-im. Texte $7=$ CTMMA 1 78, 20-26, a31-b4: “Je t'ai laissé $\frac{1}{3}$ mine 5 sicles d'argent. Je t'ai envoyé 5 sicles d'argent avec Nisašar, une servante de la maison de ton père. En tout, je t'ai laissé $\frac{1}{2}$ mine d'argent. Que Galgalliya te vende de l'orge pour une somme de 7 sicles d'argent $(. .$.$) Achète un mouton et qu'il serve de réserves de viande pour la$ servante." $\frac{1}{3}$ ma-na 5 GÍN KÜ.BABBAR, e-zi-ba-ki-im: 5 GíN KÜ.BABBAR, $N i$-ša-šar: GEME ${ }_{2} \breve{s}_{a}$ É $a-b i_{4}-k i$, ú-šé-bi $i_{4}-l a ̂-k i-i m:$ is-té-ni-iš, $\frac{1}{2}$ ma-na KÚ.BABBAR: $e-z i-b a-k i-i m($ ), ša 7 GÍN KÙ.BABBAR: ŠE-am, Gal-ga-lí-a: li-dí-na-ki-im (...) e-me-ra-am. ša-a-me-ma, ma-ás-e-er-tum, a-sú-ha-ar-tim, $l i-b i_{4}-s i$. 
couple $^{31}$. Dans la deuxième phase de l'histoire de Kunnaniya, alors que son époux est absent, Šăt-Aššur se trouve aux côtés de Kunnaniya et revendique un droit à l'héritage paternel ${ }^{32}$.

Une partie des archives privées d'Ašsur-muttabbil est vraisemblablement entreposée dans la maison du couple à Kaniš, et il envoie parfois à Kunnaniya des documents qu'elle doit ranger et conserver précieusement ${ }^{33}$. Il reproche à sa femme ses absences répétées de la maison de Kaniš, craignant ainsi qu'un voleur soit tenté de briser les sceaux et piller la chambre-forte ${ }^{34}$. Les

31. Texte 7=CTMMA 1, 78, 30-31: "Ne laisse pas Šât-Aššur mourir de faim!" Ša-âta-sur, lá rù-mi-şí, (pour la destinataire de ce passage, cf. ci-dessous) ou encore le texte 2=KTH 6, 17-20: "Laisse Sat-Assur aux bons soins de Walawala, l'esclave, on ne doit pas la livrer!" $\breve{S} a-a t-a-s ̌ u r: ~ i-n a, \breve{s} a-h a-a t$

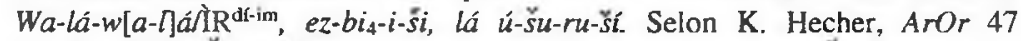
[1978], 409, Săt-A Ššur serait une servante achetée par Šu-Anum, fils d'Ennam-Sîn, BIN 4, 212=EL 213= B.Kienast, FAOS Beiheft 1, 139-140. Pour K. Nashef, WO 24 [1993], 169-170, en revanche, Šāt-Aššur serait la fille de Šallim-ahum et frère d'Ennam-A šsur. L'une et l'autre hypothèses paraissent peu vraisemblables. En effet, d'une part, Šăt-Ǎšsur, fille de Šallim-ąum, réside à Aššur d'où elle entretient une correspondance avec son mari, Aššrur-malik, fils d'Innāya (TC 3, 210) et avec d'autres marchands de Kanišs, tel

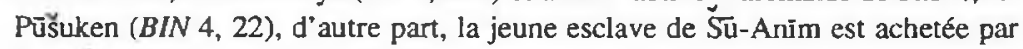
Lamăsa, fils d'Alili, et n'a rien à voir avec notre dossier.

32. Cf. ci-dessous, le texte $12=$ TTC 26.

33. Texte $1=B I N 61,3-9$ : "Concemant les tablettes que Nazi et llī-bāni t'apportent et vont te confier, partout où tu dormiras, place-les en lieu sûr!", tup-pè-e, ša Na-zi is, DINGIR-ba-ni ú-ub-lu-ni-Ri-ni-ma, i-pá-qí-du-ni-ki-ni, a-šar ta-rí-ni-li-ni, šu-uk-ni-su-nu-/ma, ša-sée-ri. D'autres tablettes sont archivées dans la maison de Kunnañya à Kaniš, appartenant sans doute à des collègues ou des membres de la famille, texte $9=C C T$ 4, 21a: "Ainsi (parle) Ennum-Aššur: (dis) à Kunnanīya et Alāhum; dis à Kunnanīya. (Concernant) les boîtes scellées et les deux tablettes scellées sous enveloppe que je t'ai laissées, là-bas, remets les boîtes scellées à Alāhum. Quant aux tablettes, qu'elles restent (là où elles sont) jusqu'à ce que mes instructions te parviennent." um-ma En-nam-a-š̀r-ma, a-na Ku-na-ni-a, ì A-là-hi-im a-na, Ku-na-ni-a, qi-bi $i_{4}-m a$ ta-ma-la-ki, ku-nu-ki ù 2 tup-pè-e, ha-ru-mu-tim, ša e-zi-ba-ku-ni, a-ma-kam ta-?ma-lá-ki, ku-nu-ki a-na, A-lá-hi-im dí-ni, ù tup-pu-ú, li-ib-ši-ú, a-dí: té-er-tí, i-lá-kà-ni.

34. La lettre 7=CTMMA 1, 78 est adressée par Aššur-muttabbil aux deux soeurs indigènes, Walhišna et Kunnanîya. Dans un premier temps, l'expéditeur s'adresse plus spécifiquement à l'aînée, mais dès la 1. 12, même si cela n'est 
inquiétudes d'Aššur-muttabbil se trouvent renforcées par la nouvelle d'émeutes au kārum de Kaniš. Alors que Kunnanìya devait le rejoindre, il semble impossible, car dangereux, de quitter les lieux ${ }^{35}$ :

"Mets-toi en route et viens. Si lorsque Kududu sera arrivé, le kärum est redevenu normal, retiens-le un jour, puis mets-toi en route (...) Si les ordres sont stricts, envoie-moi de l'huile fine et une tablette par Kududu. Et dès le kärum sera calmé, mets-toi en route et viens. Que Šamaš-takläku dorme à la porte!"

Ce court extrait montre que Kunnaniya voyage occasionnellement en Asie Mineure, mais également sans doute à Aššur où elle possède quelques biens mobiliers, bagues et fibules. Assur-muttabbil, à cours d'argent, a puisé dans les biens de sa femme à Aššur et lui cède en échange deux servantes. Mais celles-ci devaient sans doute faire partie de l'héritage de Pušu-kēn, car Buzāzu, l'un des héritiers, alors sur place à Kaniš, a pris les servantes. Aššrur-muttabbil écrit à Kunnanìya ${ }^{36}$ :

pas précisé, son interlocutrice est vraisemblablement Kunnaniya, a27-b7:

"Conserve précieusement les sceaux de la maison! Cesse de sortir sans arrêt! Surveille la maison ! (...) Jusqu'à ce que tu me voies personnellement, garde courage!" ku-nu-ki: ša É bi $i_{4}-t i m$, ša-și-ri: a-ki-dim, lá ta-ta-na-sí-i, bi $i_{4}-t a ́ m$ $[u] s-r i(.$.$) a-dí, e-ni-a: ta-me-ri-ni, li-ba-am: a-l i-b i_{4}-k i$, di-ni-i. Les pillages devaient être relativement fréquents à en croire les nombreuses mises en garde collectées dans les missives des marchands, C. Michel, "Propriétés immobilières dans les tablettes paléo-assyriennes", CRRAI 40, Leiden, 1993, à paraitre.

35. Texte $2=K T H$ 6, 9-16, 20-28: té-eb-e-ma: a-tal-ki-im, šu-ma: i-nu-mi, Ku-du-du i-li-kà-ni, ma kà-ru-um, a-na iš-ri-su, i-tù-a-ar: ú-ma-am, ǐs-té-en: $k i-i l_{5}-i-s u-m a$, té-eb-e-ma: a-tal-ki-im (...) šu-ma, a-wa-tum:da-na-a, İ.GIŠ $\mathrm{SIG}_{5}$ : ù tup-pá-am, iš-tí $K u-d u-d u$, šé-bi-lim ù a-dí-i, nu-a-ah: kà-ri-im, té-eb-a-ma: a-tal-ki-im, dUTU-ta-ak-lá<ku> a-šar, da-al-tim lu na-al. Pour ce texte, cf.K.R. Veenhof, NABU 1992/5.

36. Texte 7= CTMMA 1, 78, 12-19: ás-ta-na-ma-ma: Bu-za-zu, a-ma-ti-ki:iș-ba-at, i-țá-ba-tim: a-ma-tí-im, pu-nu-a-ma: lu-šé-ru-ni-ki-na-tí, šu-ma: e-mu-qí, e-ta-wu-ü: ma: a-ma-tim, lá ú-ša-ar: a-dí-ni-im, ku-uš-da: a-ma-tum: ku-a-a-tum. Auparavant, Aššur-muttabbil expose la situation à Walbišna, 1. 5-11: "J'ai pris une mine d"argent dans Aššur sur les propriétés de ta souer-de 
"Voilà que je ne cesse d'entendre que Buzāzu a saisi tes servantes. Tourne-toi avec bienveillance vers les servantes afin qu'il te les livre! S'il parle violemment et ainsi ne libère pas les servantes, alors, intente un procès! Les servantes sont tiennes!"

Les conflits qui ont vraisemblablement opposé Aššrur-muttabbil à ses frères et à sa soeur à la mort de Pušu-kēn opposent désormais Kunnaniya à la famille de son mari ${ }^{37}$.

\section{Kunnaniya, ruinée et abandonnée}

La deuxième phase des échanges épistolaires de Kunnaniya, caractérisée par des plaintes et inquiétudes de celle-ci vis-à-vis de sa situation, se situe sans doute après le décès d'Aššur-muttabbil. ll n'est nulle part précisé que celui-ci est effectivement mort, toutefois plusieurs allusions permettent d'évoquer cette hypothèse. Alors que Kunnanìya se trouve encore à Kaniš, un certain Aššur-taklaku lui donne les conseils suivants ${ }^{38}$ :

"Si là-bas on t'importune en quoi que ce soit, qu'une lettre de toi me parvienne, afin que j'envoie Humadašu depuis ici! Là-bas, personne ne doit te donner d'ordres, et surtout n'ouvre en aucun cas la chambre-forte de ton mari."

ses bagues ou de ses fibules-tuditrum-, et là-bas, je lui ai donné les instructions suivantes: "Contre l'argent que j'ai pris, que les deux servantes soient tiennes!"

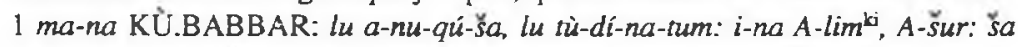
a-ha-tí-ki: al-qé-ú: a-ma-tum, ki-lá-al-ta-ma: lu ku-a-a-tum. Pour la lecture de ce texte, cf. K. Hecher, $N A B U$ 1990)139, et pour le terme tuditum, H. Klein, ZA 73 [1983], 255.

37. Différents jugement et procès-verbaux indiquent des règlements de comptes entre As̉sur-muttabbil et le reste de sa famille comme $T C 1,79=E L 11$ où Asšur-muttabbil s'est fait rembourser une dette impayée envers son père de 40 mines d'argent.

38. Texte $10=B I N 6,17,3-10$, su-ma a-ma-kam li-bi $-k i$, mi-ma ú-lá-mu-nu té-er-tí-ki, a-șé-ri-a li-li-kam-ma, Hu-ma-da-šu a-na-nu-um, lá-aț-ru-dam a-ma-kam, ma-ma-an e $\hat{u}$-wa-ir-ki-ma, ma-ak-na-kam sa mu-tí-ki, e ta-áp-tí-a. 
Il est probable que la famille d'Ašsur-muttabbil tente de mettre la main sur des documents relatifs à la succession de Pūsu-kēn, ou encore liés à des opérations financières menées par la firme familiale. D'autre part, Kunnaniya se plaint que le loyer d'une maison qui, selon elle, devait lui revenir, ou tout du moins aux fils d'Assur-muttabbil, a en fait été perçu par Püšu-kēn, certainement le fils Buzăzu et petit-fills de Püšu-kēn ${ }^{39}$. Les inquiétudes de Kunnaniya, qui se retrouve seule, confrontée à une puissante famille de marchand assyrien, apparaissent également dans une missive que lui envoie sa soeur, Walhišna (8).

Néanmoins, alors que Kunnaniya a quitté Kaniš, ses attaques virulentes ne portent plus sur la famille de son époux, mais contre deux femmes et un homme aux noms anatoliens, qui appartiennent vraisemblablement à sa propre famille. Săt-Aššur, certainement enfant dans les lettres envoyées par Aššur-muttabbil à Kunnanīya, apparaît plus âgée dans ces quelques documents.

Contrairement aux autres lettres de sa correspondance, les quatre derniers messages émanent de Kunaniya ${ }^{40}$. Deux hypothèses s'imposent quant à sa résidence. On peit imaginer que Kunnanìya s'est rendue à Aššur pour régler les affaires de la succession de son époux, mais il est également possible, bien que cela paraisse moins probable, qu'elle soit temporairement installée dans une autre localité anatolienne d'où elle écrit à Kaniš. Quoiqu'il en soit, elle se trouve aux côtés d'Aššur-malik, peut-être l'un de ses filss ${ }^{41}$, et de

39. Texte 11= $A K T$ 1, 14, 18-24: "Eh bien, Pứsu-kēn a pris le loyer de la maison à la place des fils d'Aššrur-muttabbil. Ce serait bien ainsi! Au lieu de prendre moi-même le loyer de la maison, maintenant Pǔsu-kēn vient de le prendre."

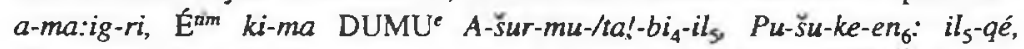
ki-a-am: da-mi-iq: ki-ma, a-na-ku: ig-ri bi $i_{4}$-tim, lá-qá-im a-ni Pu-šu-ke-en, $i l_{5}$-té-qé. Les fils d'Aššur-muttabbil ne sont malheureusement nullement nommés.

40. Lettres $11,12,13$ et 14 . Cette dernière missive adressée par Kunnañya à Kubinabsu (CCT 6,7b) n'est cependant pas exploitable car elle ne mentionne aucun personnage connu et elle est trop fragmentaire.

41. Le texte 13= KTH 5 est expédié à la fois par Kunnanīya et par Aššur-malik (1.24-26). Notons que, parmi les très nombreux homonymes d'Aššr-malik, il 
Šăt-Aššur, peut-être sa fille ${ }^{42}$. En son absence, Kunnanīya a confié sa demeure kanisite et ses biens mobiliers à Asu(w)elka, sans doute sa soeur aînée, qui avait entreposé chez elle des denrées alimentaires ${ }^{43}$, ainsi qu'à Adue. Cette dernière, vraisemblablement épouse de Šesșur, peut-être un frère de Kunnanīya, habite alors avec son mari la maison de Kunnaniya ${ }^{44}$. Non seulement Kunaniya n'a pu percevoir le loyer de sa maison dont son beau-frère, Buzāzu, s'est emparé (11), mais de plus, son mobilier et toutes ses biens à Kaniš ont été pillés ${ }^{45}$. Šāt-Aššur se plaint amèrement auprès d'Adue d'avoir été ainsi dépossédée par la famille de son père ${ }^{46}$ :

existe un Aššr-malik, fils d'Ašsur-muttabbil, $T C 2,75=A L 264,8-9$ ou $E L$ $144,4-5$.

42. Kunnanīya et Săt-Aššur rédigent ensemble la lettre $12=\operatorname{TTC} 26=\mathrm{C}$. Michel, RA 80 [1986], 127-128.

43. Texte $12=\operatorname{TTC} 26,5-18$ : "Lorsque je suis arrivée à Kaniš, tes pains de bière avaient été placés en dépôt. Aussi, je t'avais confié du mobilier en disant: "Lorsque tu auras sorti tes "pains de bière", alors scelle le dépôt !" Ne t"ai-je point dit ceci: "Ne me le fais pas regretter!" Eh bien aujourd"hui, je le regrette! Qu'ils disparaissent là où mes caisses ont disparues!fg $i-n u-m e: a-n a K \grave{a}-n i-i \grave{s}$, e-ru-ba-ni: i-na hu-ur-ší-im, ba-pi-ir-ki: iš-ši-ki-in, ú ú-nu-tám áp-qí-da-/ki-im, um-ma a-na-ku-ma: ki-ma, ba-pi-ir-ki: tù-šé-și-ni, ú hu-ur-ša-am: ku-un-ki, lá-aq-bi $i_{4}-a-k i-i m$ um-ma, a-na-ku-ma a-na lam-ni-šu, lá tù-ta-ri-ni, us-ma-am lam-ni-šu, a-tù-wa-ar e-ma, ta-ma-la-ku-a: ú šu-nu, li-ih-li-qú-ma. Asu (w)elka est la femme d'Ispunuman, TC 3,254, 5.

44. Sessur intervient dans le contrat $B I N 6,226,4$ parmi les acheteurs d'un esclave, et il est l'un des témoins du document Neşr C 10 (communication de K.R. Veenhof). Le lien matrimonial entre Sesur et Adue peut être déduit de la lettre 11= AKT 1, 14. Cette missive expédiée à Šesșur est en fait adressée également à Adue. Kunnaniya y mentionne une enfant qui serait la fille du couple L. 24-29: "Dis à Adue. Eh bien, on va amener ta fille, aime-la! Dis à Sēșur. Eh bien, on va amener ta fille, là-bas aime-la!" $a-n a A-d u-e, ~ q i$-bi $i_{4}-m a$ a-ma: me-er-at-ki, i-ra-dí-ú-nim ra-mì-i-š́, a-na Šé-sú-ur! (IR) qí-bi $i_{4}-m a, a-m a$ me-er-at-kà i-ma-dí-ú-/nim, a-ma-kam ra-a-am-š́.

45. Texte $11=A K T$ 1, 14, 32-36: "Dis à Adue. Eh bien, là-bas, il n'y a plus rien de la maison et du mobilier, et c'est de ta faute!" a-na A-du-e qi-bi $i_{4}-m a, a-m a$ :

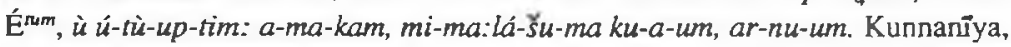
pour sa part, refuse de payer un loyer pour la maison qu'elle occupe, d'autant plus qu'elle n'est point la seule à y vivre $(13,19-24)$.

46. Texte $12=\operatorname{TTC} 26,19-30:$ a-na A-du-e qi-bi $i_{4}-m a$, um-ma కa-al-a-sur-ma $a-n a-k u$, a-na um-mi-a ás-ta-na-kà-ki, um-ma <<ma>> a-na-ku-ma, a-lá: u-nu-tù î-na, Kà-ni-iš i-ba-צ̌́, a-tí: pá-ta-a-tí-ma, tù-ùh-ta-li-qí, mi-šu-um sa-ah-ar-ku-ma, ú bi $i_{4}$-tám ša a-bi ${ }_{4}-a$, lá ú-ga-ra-ma i-na, i-ga-ri-im 
«Dis à Adue: ainsi (parle) Šăt-Aššur. Moi, alors que je n'ai cessé de te considérer comme ma mère, voici donc ce que j'ai à te dire: "Excepté le mobilier qui se trouvait dans Kaniš, toi tu (étais) la gardienne (du mobilier), et de ce fait, tu m'a ruinée!" Pourquoi donc suis-je trop jeune que je ne puisse faire un procès à la famille de mon père? Mon clou (est) pourtant dans le mur!

Désespérée et se sentant abandonnée de tous, Kunnanīya écrit une lettre pathétique à Asu(w)elka ${ }^{47}$ :

"Si tu (es) ma soeur et si tu m'aimes véritablement (écoute-moi)! Je n'en peux plus, je vais mourir! Je'nai pas d'issue! Auparavant, mon message ne t'était pas parvenu, mais aujourd'hui ma lettre t'est (nécessairement) parvenue! Alors, toi, prononce un seul mot et je viendrai!"

Ce petit dossier épistolaire retrace donc les déboires d'une femme, heureuse au début de sa vie, puis abandonnée et aux prises avec sa puissante belle-famille et avec sa propre famille. Son intrérêt tout particulier réside dans le lien qui unit Kunnaniya à la famille de pūšu-kēn, le plus célèbre marchand de Kaniš. Mais il s'agit également là d'une des rares illustrations de la vie d'une femme anatolienne de Kaniš. En effet, la majorité de la documentation féminine paléo-assyrienne reflète généralement les activités des femmes assyriennes intallées à Aššur, qui écrivent à leur mari et leurs proches partis commercer en Asie mineure.

sí-kà-tám-ma K. Nashef, WO 11 [1994] 170, note 11 traduit ainsi les 1.20-22: "Je t'ai sans cesse placée au service de ma mère".

47. Texte 13=KTH 5, 3-12: \$u-ma: a-ha-tí: a-tí, ma ki-na-tí-ma ta-ra-i-mi-/ni, lá al-té-e: a!-mu-wa-lat-ma, qá-qí-ri-i: lá-šu, i-na pá-ni-tim, té-er-tí: lá i-li-kà-kum, u-ma-am: na-ás-pè-er-tí, i-li-kà-kum: ù a-tí, a-wa-tám iš-té-ento qá-ri-ba-ma ú tá-tal-kam-/ma. 